\title{
CONDITIONAL MAXIMUM LIKELIHOOD FREQUENCY ESTIMATION FOR STAGGERED MODULATIONS
}

\author{
Jaume Riba, Gregori Vázquez and Sergio Calvo \\ Department of Signal Theory and Communications, \\ UPC. Campus Nord. Edifici D-5, c/ Gran Capità. s/n, 08034 Barcelona, Spain, \\ Email: \{jriba,gregori\}@gps.tsc.upc.es
}

\begin{abstract}
The use of spectrally efficient continuous phase modulations for mobile communications may lead to a serious performance degradation of the classical frequency error detectors (FEDs) due to the presence of self-noise. This contribution presents a new statistically efficient frequency estimation algorithm for staggered modulations. The cancellation of the self-noise is accomplished by the use of the Conditional ML principle, well known in the context of array processing, as an alternative to the Unconditional ML, typically applied in the communications field. The paper also provides a new Cramer Rao Bound (CRB) which is more accurate than the so-called Modified CRB (MCRB) extensively applied to synchronization problems.
\end{abstract}

\section{INTRODUCTION}

A Frequency Error Detector (FED) is the basic component of an automatic frequency loop. This kind of loop, either in analog or digital form, is usually employed for the purpose of carrier frequency synchronization in digital communications. Different types of FEDs have been proposed in the last few years, and basically, there may be classified in four types: quadricorrelators, dual-filter detectors (DFD), rotational detectors and ML-based detectors. The reader is referred to the F.M. Gardner report [1] and references therein, and to D'Andrea et al. papers [2][3] for a further study of ML-based methods and the Cramer-Rao bounds associated to this problem.

Frequency detectors based on the Maximum Likelihood (ML) formulation are important in that they are expected to provide good performance against noise. However, in the case of digitally modulated signals, the assumption of low signal-to-noise ratio (SNR) is usually required in order to avoid the mathematical difficulties associated with the rigorous application of the ML principle. To assume that the SNR is low has the consequence that the final structure of the estimator ignores the effect of the so-called self-noise. It is demonstrated in [1] that self-noise-free operation can be achieved with non-staggered signals (when the loop is in the steady state) provided that two mild restrictions are satisfied, i.e., the link impulse response $g(t)$ is Nyquist $(g(i T)=0, i \neq 0)$ and the Fourier Transform

This work has been supported by PRONTIC/CICYT TIC95-1022-C05-01, PRONTIC/CICYT TIC96-0500-C10-01 and CIRIT/Generalitat de Catalunya 1996SGR-0096. of the transmitted pulse has linear phase. However, in the case of staggered signals, the more stringent requirement that $g(i T / 2)=0$ at $i \neq 0$ should be met for the cancellation of the self-noise, which is too restrictive for practical applications.

This paper presents a new FED solution for the case of staggered signals which has the property that the self-noise is totally cancelled without the necessity of the above mentioned restrictive condition. The key point for the derivation of the new solution is the use of a different method for the application of the ML principle, which does not require the assumption of low SNR. In this sense, one of the goals of the present contribution is to show that the conclusions derived in the literature concerning the requirements for the self-noise-free condition can not be considered as general conclusions about the frequency estimation problem. Instead, it is the assumption of low-SNR required in the classical derivation what leads to these stringent requirements. While in the field of array signal processing, both Conditional ML (CML) and Unconditional ML (UML) methods [4] have been studied and successfully applied to different problems, in the field of digital communications only the UML method has been classically employed. A possible reason for that is that most synchronization algorithms for digitally modulated signals have been obtained by formulating the problem in the continuous time domain, and their discrete counterparts have been derived by a direct translation of them. Then, the question arises to whether the CML method can be applied directly to the sampled digital modulations, and whether the obtained results will differ or not with respect to the classical ones derived from the UML method. This question is addressed in the present contribution where both classical and new algorithms are derived via the CML principle by formulating the problem directly in the discrete time domain.

The present contribution makes use of the high amount of research effort on the CML in the field of array processing, by importing all those rich results to the frequency estimation problem of digitally modulated signals. Specifically, on the one hand, the final structure of the FEDs is based directly on the general equations for the gradient and Hessian derived by Viberg et al. [5]. On the other hand, the general expression for the Cramer-Rao Bound (CRB) for the CML method derived in [4] is used to derive a more accurate performance bound than the so-called Modified CRB (MCRB) derived in [3]. 


\section{BACKGROUND}

The derivation of the algorithm is done from the general class of linear problems according to the following expression:

$$
\mathbf{Y}=\mathbf{A}_{p}(\boldsymbol{\theta}) \mathbf{X}+\mathbf{W}
$$

where the $B x M$ matrix $\mathbf{Y}$ denotes the observation matrix composed by $M$ observation vectors y $(i)(i=0,1,2, \ldots, M-$ 1) of length $B$ samples each, the $N x B$ matrix $\mathbf{X}$ the transmitted data symbols, the $N x M$ modulation dependent signal model transfer matrix $\mathbf{A}_{p}(\boldsymbol{\theta})$, and the $B x M$ noise matrix $W$ the AWGN term with scalar element by element mean power $\sigma_{w}^{2}$. The CML estimation of parameter vector $\theta$ is obtained by the minimization of the following cost function:

$$
L_{C M L}(\theta)=\operatorname{tr}\left(\mathbf{P}_{A}^{\perp} \widehat{\mathbf{R}}\right)
$$

where the observation data samples autocorrelation matrix $\widehat{\mathbf{R}}=\mathbf{Y} \mathbf{Y}^{H} / M$ is projected into the orthogonal signal space by mean of the orthogonal projection matrix $\mathbf{P}_{A}^{\perp}=$ $\mathbf{I}-\mathbf{A}_{p} \mathbf{A}_{p}^{\sharp}=\mathbf{I}-\mathbf{A}_{p}\left(\mathbf{A}_{p}^{H} \mathbf{A}_{p}\right)^{-1} \mathbf{A}_{p}^{H}$. Under these conditions, Stoica and Nehorai [4] derived the Cramer-Rao bound (CRB) for the CML estimator in the context of linear array theory. On the other hand, Viberg, Ottersten and Kailath [5] derived the gradient and Hessian of the CML cost function (2). For the single parameter case (as happens in the problem of frequency estimation) for which $\theta=\Omega 1$, the CRB, gradient and Hessian equations may be manipulated to yield:

$$
\begin{gathered}
C R B_{C M L}(\Omega)=\left(\frac{2 B}{\sigma_{w}^{2}} \operatorname{Re}\left[\operatorname{tr}\left(\mathbf{D}^{H} \mathbf{P}_{A}^{\perp} \mathbf{D} \widehat{\Gamma}\right)\right]\right)^{-1} \\
\widehat{\Omega}_{C M L} \approx \bar{\Omega}-\frac{1}{E[H(\Omega)]} g(\bar{\Omega}) \\
g(\bar{\Omega})=-2 \operatorname{Re}\left[\operatorname{tr}\left(\mathbf{A}_{p}^{\sharp} \widehat{\mathbf{R}} \mathbf{P}_{A}^{\perp} \mathbf{D}\right)\right] \\
E[H(\Omega)]=2 \operatorname{Re}\left[\operatorname{tr}\left(\mathbf{D}^{H} \mathbf{P}_{A}^{\perp} \mathbf{D} \mathbf{\Gamma}\right)\right]
\end{gathered}
$$

where $\widehat{\Gamma}=\mathbf{X X}^{H} / M$ is the autocorrelation matrix obtained from the symbol matrix $\mathbf{X}$ and matrix $\mathbf{D}(\Omega)=\frac{\partial}{\partial \Omega} \mathbf{A}_{p}(\Omega)$ The following sections constitute a summary of the approach followed in [6].

\section{FREQUENCY ESTIMATION FOR NON-STAGGERED MODULATIONS}

Let's consider the signal model for a non-staggered linear digital modulation (e.g. MPSK, MQAM,...) affected by a frequency error $\Omega$. The received signal in an AWGN channel can be modeled by eq. (1). The received data vector signal model of length $B$ samples can be written as follows:

$$
\mathbf{y}=[y(0), y(1), \ldots, y(B-1)]^{T}=\mathbf{A}_{p}(\Omega) \mathbf{x}+\mathbf{w}
$$

where $\mathbf{A}_{p}(\Omega)$ has the conventional convolution structure of the pulse shaping filter:

$$
\mathbf{A}_{p}(\Omega)=\left(\begin{array}{llll}
\mathbf{a}_{p}(\Omega) & \mathbf{0}_{N_{s s}} & \cdots & \vdots \\
\mathbf{0}_{N_{s s}} & \mathbf{a}_{p}(\Omega) & \ldots & \vdots \\
\vdots & \mathbf{0}_{N_{s s}} & \ddots & \mathbf{0}_{N_{s s}} \\
\vdots & \vdots & \mathbf{0}_{N_{s s}} & \mathbf{a}_{p}(\Omega)
\end{array}\right)
$$

being $\mathbf{a}_{p}(\Omega)$ the pulse shape response $\mathbf{p}$ of length $2 K+1$ symbols sampled at $N_{s s}$ samples per symbol affected by the frequency offset $\mathbf{a}(\Omega)$, that is:

$$
\begin{aligned}
\mathbf{a}_{p}(\Omega) & =\mathbf{p} \odot \mathbf{a}(\Omega) \\
\mathbf{p} & =\left[p(0), p(1), \ldots p\left((2 K+1) N_{s s}-1\right)\right]^{T} \\
\mathbf{a}(\Omega) & =\left[1, e^{j \Omega}, e^{j 2 \Omega}, \ldots, e^{j\left((2 K+1) N_{s s}-1\right) \Omega}\right]^{T}
\end{aligned}
$$

and being $\mathrm{x}=\left[x(0), x(1), \ldots, x\left(N_{s}-1\right)\right]^{T}$ the information symbol vector which includes the carrier phase evolution sampled at one sample per symbol:

$$
x(n)=A e^{j\left(\phi+n N_{s s} \Omega\right)} s(n)
$$

The log-CML cost function (2) becomes:

$$
\begin{gathered}
L_{C M L}(\Omega)=\operatorname{tr}\left(\mathbf{P}_{A}^{\frac{1}{4}} \mathbf{y} \mathbf{y}^{H}\right)=\text { const. }-\frac{N_{s}}{E_{p}} \widehat{S}_{p}(\Omega) \\
\widehat{S}_{p}(\Omega)=\frac{E_{p}}{N_{s}}\left(\mathbf{A}_{p}^{\sharp}(\Omega) \mathbf{y}\right)^{H}\left(\mathbf{A}_{p}^{H}(\Omega) \mathbf{y}\right)
\end{gathered}
$$

where $E_{p}$ is the pulse energy. For ISI-free pulse shaping it holds that $\mathbf{A}_{p}^{H}(\Omega) \mathbf{A}_{p}(\Omega)=E_{p} \mathbf{I}$ and the cost function to be maximized becomes:

$$
\widehat{S}_{p}(\Omega)=\frac{1}{N_{s}}\left\|\mathbf{A}_{p}^{H}(\Omega) \mathbf{y}\right\|^{2}
$$

or, in other words, it is necessary to maximize in $\Omega$ the mean power of the matched filter $\mathbf{a}_{p}(\Omega)$ output decimated at one sample per symbol which is more clearly represented by the following expression:

$$
\widehat{S}_{p}(\Omega)=\frac{1}{N_{s}} \mathbf{a}^{H}(\Omega)\left[\widehat{\mathbf{R}}_{s n c} \odot \mathbf{p} \mathbf{p}^{H}\right] \mathbf{a}(\Omega)
$$

or, in other words, the periodogram over the synchronous autocorrelation matrix $\widehat{\mathbf{R}}_{s n c}=\sum_{i=0}^{N_{s}-1} \mathbf{y}_{i} \mathbf{y}_{i}^{H}$ of the strobe by strobe partially overlapped received data $\mathbf{y}_{i}$, weighted by the outer product of the matched filter $\mathbf{p}$ response, with:

$$
\mathbf{y}_{i}=\left[y\left(i N_{s s}\right), y\left(i N_{s s}+1\right), \ldots, y\left(i N_{s s}+(2 K+1) N_{s s}-1\right)\right]
$$

Finally, the solution update recursion given by eq. (4) is derived from the gradient expression in eq. (5) and the Hessian of the CML cost function in eq. (6). For a given prior $\bar{\Omega}$ close the CML solution, the gradient becomes:

$$
g(\bar{\Omega})=-\frac{2}{E_{p}} \sum_{i=0}^{N_{s}-1} \operatorname{Im}\left[\left(\mathbf{a}_{p}^{H}(\bar{\Omega}) \mathbf{y}_{i}\right)\left(\mathbf{b}_{1}^{H}(\bar{\Omega}) \mathbf{y}_{i}\right)^{*}\right]
$$




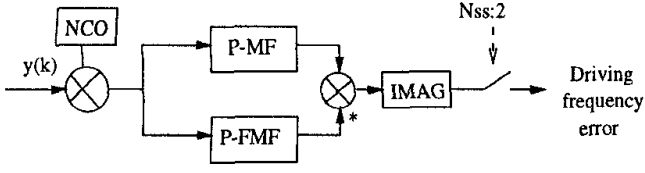

Figure 1: Structure of the FED. The obtained FED employing the CML approach resembles that of classical Gardner's FED, but with different design of the two filters.

where $\mathbf{b}_{1}=-j\left(\mathbf{I}-\frac{1}{E_{p}} \mathbf{a}_{p}(\bar{\Omega}) \mathbf{a}_{p}^{H}(\bar{\Omega})\right) \frac{\partial}{\partial \Omega} \mathbf{a}_{p}(\Omega)_{\Omega=\bar{\Omega}}$. This result agrees with that given by Gardner [1] where the estimation update is driven by the product of two FIR filter outputs, the matched filter (MF) and the frequency matched filter (FMF), as depicted in figure 1. On the other hand, the Hessian given in eq. (6) leads to:

$$
E[H(\Omega)]_{\Omega=\bar{\Omega}}=2 \beta_{p} P_{T}
$$

where $P_{T}=E\left[\left|\mathbf{a}_{p}^{H}(\bar{\Omega}) \mathbf{y}_{i}\right|^{2}\right]$ is the mean power at the output of the MF and $\beta_{p}$ a measure of the pulse energy timespread $\beta_{p}=E_{p}\left[\sum_{k} k^{2} \epsilon_{p}(k)-\left(\sum_{k} k \epsilon_{p}(k)\right)^{2}\right]$ for $\epsilon_{p}(k)=$ $|p(k)|^{2} / E_{p}$. The asymptotic CRB (eq. (3)) for the frequency estimation is given by:

$$
C R B_{C M L}(\Omega)=\frac{1}{2 N_{s} S N R \beta_{p}}
$$

which agrees with the MCRB given by Moeneclaey et al. [7] and D'Andrea et al. [3].

\section{EXTENSION FOR STAGGERED MODULATIONS}

Staggered modulations (e.g. OQPSK, MSK,...) consist in the modulation of two orthogonal subcarriers with a constant time-delay between them. There are two reasons for using this modulation structure. The first one is to achieve an almost continuous phase time evolution (in fact, MSK is a particular case of CPFSK) and the second is to ensure a more constant signal envelope time evolution for improving the performance in front of non-linearities in the high power amplifiers.

The signal model given in eq. (7) for non-staggered modulations is also valid for the staggered modulations with small changes. Basically, the unique difference is introduced by the constant time-offset between the orthogonal subcarriers. The most typical offset is half a symbol period and this is reflected in the transfer matrix $\mathbf{A}_{p}(\Omega)$ as follows:

$$
\mathbf{A}_{p}(\Omega)=\left(\begin{array}{llll}
\mathbf{a}_{p}(\Omega) & \mathbf{0}_{N_{s s} / 2} & \cdots & \vdots \\
\mathbf{0}_{N_{s s} / 2} & \mathbf{a}_{p}(\Omega) & \cdots & \vdots \\
\vdots & \mathbf{0}_{N_{s s} / 2} & \ddots & \mathbf{0}_{N_{s s} / 2} \\
\vdots & \vdots & \mathbf{0}_{N_{s o / 2}} & \mathbf{a}_{p}(\Omega)
\end{array}\right)
$$

Note that the ISI-free condition obtained for non-staggered modulations no longer holds (i.e. $\left.\mathbf{A}_{p}^{H}(\Omega) \mathbf{A}_{p}(\Omega) \neq E_{p} \mathbf{I}\right)$. The modification of the transfer matrix $\mathbf{A}_{p}(\Omega)$ also requires the extension of the symbol vector $\mathbf{x}$ to the modified one $\mathrm{x}=\left[x(0), x(1), \ldots, x\left(2 N_{s}-1\right)\right]^{T}$ where now the length of the vector becomes $2 N_{s}$ instead of $N_{s}$, with even and odd modified symbols given by:

$$
\begin{gathered}
x(2 n)=A e^{j\left(\phi+n N_{s s} \Omega\right)} \operatorname{Re}[s(n)] \\
x(2 n+1)=A e^{j\left(\phi+(n+1 / 2) N_{s s} \Omega\right)} j \operatorname{Im}[s(n)]
\end{gathered}
$$

The new CML cost function is, once again, given by eq. (14), but now, $\mathbf{A}_{p}^{\sharp}(\Omega) \neq 1 / E_{p} \mathbf{A}_{p}^{H}(\Omega)$. Note that, since a CML cost function is employed, the perfect suppression of the pattern noise is ensured, because the term $A_{p}^{\sharp}(\Omega) y$ extracts the minimum mean square error estimate of the symbol information vector $\mathbf{x}$, which is used to remove the symbol information at the matched filter output $\mathbf{A}_{p}^{H}(\Omega) \mathbf{y}$. This property applies for the frequency estimation in both, non-staggered and staggered modulations, and it is the fundamental difference with the UML based algorithms.

For the computation of the small error gradient let's recall from eq. (5) that:

$$
g(\bar{\Omega})=-2 \operatorname{Re}\left[\operatorname{tr}\left(\mathbf{A}_{p}^{\sharp} \mathbf{y} \mathbf{y}^{H} \mathbf{P} \frac{\perp}{A} \mathbf{D}\right)\right]
$$

It is useful to focus on the discussion of the first term $\mathbf{A}_{p}^{\sharp} \mathbf{y}$. The pseudo-inverse matrix is performing as a zero-forcing matrix. For the asymptotic case $B \longrightarrow \infty$, the CML estimation will become unbiased and the central rows of matrix $\mathbf{A}_{p}^{\sharp}$ will only differ in a time-delay. This property holds if the transfer matrix $\mathbf{A}_{p}$ is full-rank or, in other words, if the modulation has an excess of band larger than the required by the sampling rate to ensure a non-zero discrete spectrum. This condition applies for OQPSK, MSK, among many others, but it could not be satisfied for perfect band limited modulations. Under this constraint, the computation of the frequency error gradient is straightforward. If we define the pseudo-matched filter P-MF as $\mathbf{p}_{\text {asym }}$ and the frequency pseudo-matched filter P-FMF as $\mathbf{b}_{2}$, the gradient estimate will follow the same structure as for the non-staggered modulations (eq.(18)), that is:

$$
g(\bar{\Omega})=-2 \operatorname{Im}\left[\left(\mathbf{p}_{a s y m}^{H} \mathbf{y}_{i}\right)\left(\mathbf{b}_{2}^{H} \mathbf{y}_{i}\right)^{*}\right]
$$

where:

$$
\begin{gathered}
\mathbf{p}_{\text {asym }}=\text { central row } \lim _{B \rightarrow \infty} \mathbf{A}_{p}^{\sharp} \\
\mathbf{b}_{2}=\text { central row } \lim _{B \rightarrow \infty}-j \mathbf{D}^{H} \mathbf{P}_{A}^{\perp}
\end{gathered}
$$

The estimation scheme is, once again, given by figure 1 . Finally, the CRB is also derived and it follows the same expression given for non-staggered modulation in eq. (20) where parameter $\beta_{p}$ becomes $\beta_{p}^{\text {stag }}$ is given:

$$
\beta_{p}^{s t a g}=\mathbf{d}^{H} \mathbf{P}_{A}^{\perp} \mathbf{d}
$$

where

$$
\mathbf{d}=\text { central row } \lim _{B \rightarrow \infty} \mathbf{D}
$$

\section{SIMULATION RESULTS}

This section illustrates the philosophy and performance of the presented design procedure for the case of a MSK signal with $N_{s s}=8$ samples per symbol, which is interpreted 
as a staggered signal with cosenoidal pulse shaping. Figure 2 shows the impulse response of the MF and FMF filters. This is the classical result obtained by applying the UML method. It is seen that the output of these filters to a single pulse is not zero at some sampling instants different from the strobe, and this is the cause of the resulting self-noise of the UML method. Figure 3 shows the same results as
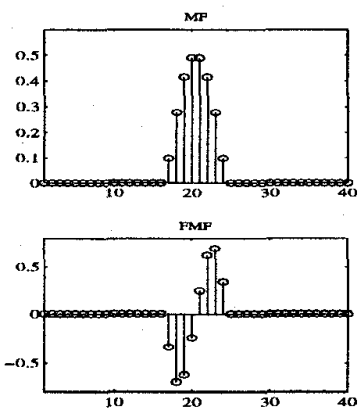

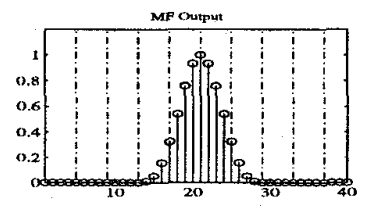

FMI coutpow

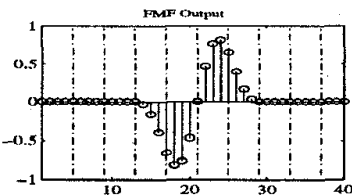

Figure 2: UML method. MF and FMF filters for MSK along with their response to a single symbol.

figure 2 in the case of adopting the proposed CML design procedure. It is seen that both P-MF and P-FMF filters have changed with respect to the MF and FMF pulses in such a way that the output of these filters to a single cosenoidal pulse is now zero at the sampling instants, except for the strobe. For that reason, the new solution does not exhibit self-noise at the steady state. Finally, figure 4 shows
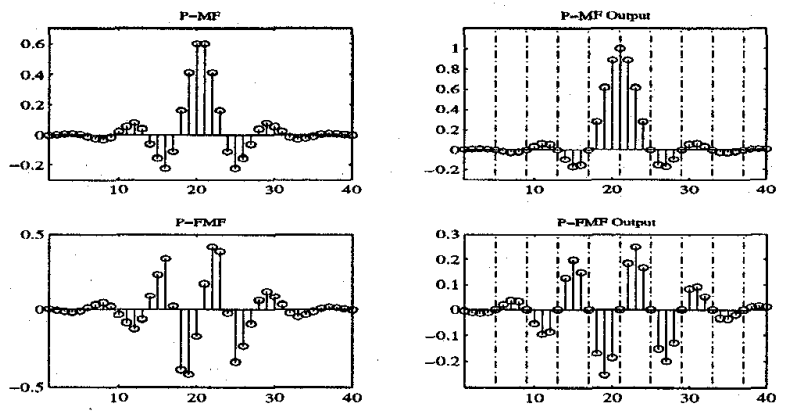

Figure 3: CML method. P-MF and P-FMF filters for MSK along with their response to a single symbol.

the variance of the frequency estimate normalized to the effective observation time of the FED loop. It is seen that for moderate to high energy per symbol to noise spectral density $\left(E_{s} / N_{o}\right)$, the main source of performance degradation of the classical UML method is due to the presence of self-noise. For that reason the good performance predicted by the MCRB is never attained. On the contrary, the CML estimator attains the more accurate CRB derived in this paper. The only price for this improved behaviour is a little degradation of the CML method with respect to the UML method in the lower range of $E_{s} / N_{o}$.

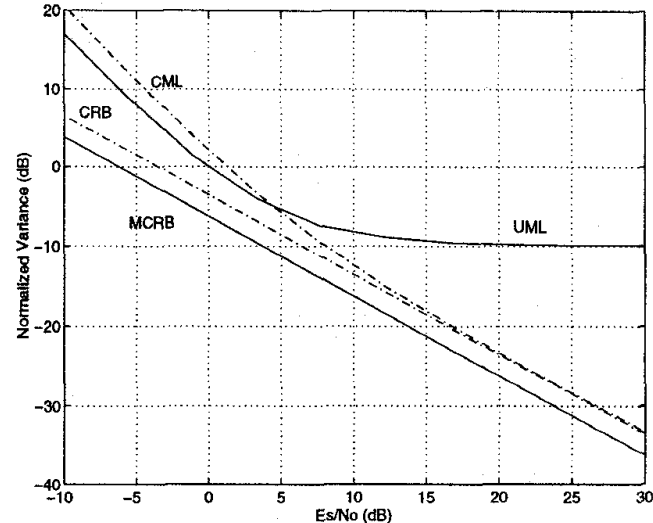

Figure 4: Normalized steady state variance of the estimated frequency for the UML and CML methods, along with the CRB (CML) and the MCRB (UML).

\section{CONCLUSIONS}

The CML method is applied in this paper to a synchronization problem, as an alternative to the classical UML procedure typically applied in the field of digital communications. The main advantage of the new approach is that the self-noise can be totally cancelled for those typical cases (e.g. staggered signals) for which the classical approach leads to this undesirable effect. The new approach does not require any statistical assumption about the symbols, and the classical low-SNR assumption is not required as happened in the UML case. Therefore, it constitutes a better founded way of applying the ML principle for deriving existing and new solutions. The future work will address the extension of the method to the GMSK format, better suited to mobile communications.

\section{REFERENCES}

[1] F. M. Gardner. Frequency detectors for digital demodulators via Maximum-Likelihood derivation. Final Report: Part II, ESTEC No. 8022/88/NL/DG, ESA, Jun., 1990.

[2] A. D'Andrea and U. Mengali. "Noise performance of two frequency-error detectors derived from maximum likelihood estimation methods". IEEE Tr. Comm., vol. 42 (NO. 2/3/4): pp. 793-802, Feb/Mar/Aprl 1994.

[3] A. D'Andrea, U. Mengali, and R. Reggiannini. "The modified Cramer-Rao bound and its application to synchronization problems". IEEE Tr. Comm., vol. 42 (NO. 2/3/4): pp. 1391-1399, Feb/Mar/Apr 1994.

[4] P. Stoica and A. Nehorai. "Performance study of conditional and unconditional direction-of-arrival estimati-
on". IEEE Tr. ASSP, vol. 38: pp. 1783-1795, Oct. 1990.

[5] M. Viberg, B. Ottersten, and T. Kailath. "Detection and estimation in sensor arrays using weighted subspace fitting". IEEE Tr. Sig. Pro., vol. 39(11): pp. 2436-2449, Nov. 1991

[6] J. Riba. A Bayesian Approach to Joint Frequency and Time-Delay Estimation. PhD thesis, UPC (Barcelona), 1997.

[7] M. Moeneclaey and I. Bruyland. "The joint carrier and symbol synchronizability of continuous phase modulated waveforms". In Conf. Rec. ICC'86, volume 2, 1986. paper 31.5. 